\title{
Lesional diagnostic approach of common dermatoses in children at the University Clinics of Kinshasa - Democratic Republic of Congo
}

\section{Lydie Joelle Seudjip Nono', Hugues D. Adegbidi², Adama Traore ${ }^{3}$, Paulo Bunga Muntu4}

${ }^{1}$ Department of Dermatology and Venereolology, Kinshasa University Clinics, Democratic Republic of Congo, ${ }^{2}$ Department of Dermatology and Venereolology, University Hospital Center, Cotonou, Benin, ${ }^{3}$ Department of Dermatology and Venereolology, Yalgado Ouédraogo University Hospital Center in Ouagadougou, Burkina Faso, ${ }^{4}$ Department of Pediatrics, Kinshasa University Clinics, Democratic Republic of Congo

Corresponding author: Dr. Lydie Joelle Seudjip Nono, E-mail: seupiziemi@gmail.com

\begin{abstract}
Background: Several authors approach epidemiological studies on dermatoses using an etiological diagnostic approach; the Willaniste school being mostly reserved for learners of dermatology and/or non-dermatologist doctors. The objective of this study is to determine the profile of dermatoses in children using exclusively the type of elementary lesion. Material and methods: In a retrospective, analytical and descriptive study, the data of children with dermatoses followed in the Dermatology Service of the University Clinics of Kinshasa between June 1, 2009 and December 31, were collected. The parameters of interest included epidemiological and clinical characteristics. Results: The hospital frequency of dermatoses in children (DC) according to the lesional diagnostic approach is $40.89 \%$ (818/1994). Their median age was 60 months (QEI 60-65.9) with a female predominance (55.7\%, sex ratio of $1.25 / 1$ ). There were more infants (30.6\%). DC predominated in the dry season (54\%). The entangled (21.02\%) and vesicular (20.29\%) lesional types were the most numerous, with ringworm of the scalp (31.9\%) and atopic dermatitis $(54.2 \%)$ respectively. The papular, erythematous, tumor and entangled lesion types were related to sex and age; pustular and tumor exclusively related to the season, in a statistically significant way $(\mathrm{p}<0.05)$. Taken as a whole, the most frequent dermatoses were atopic dermatitis (11\%), prurigo strophulus (10.8\%), impetigo (7.4\%), Tinea capitis (6.7\%) and scabiosis $(6.4 \%)$. Conclusion: The importance of this work lies in the interest of taking into account the two approaches, lesional and etiological of dermatoses, for their global management and research studies. Indeed, the lesional approach, also important in the study of dermatoses, appears to our knowledge to be poor in references in the literature.
\end{abstract}

Key words: Dermatoses; Children; Lesional diagnostic; Kinshasa

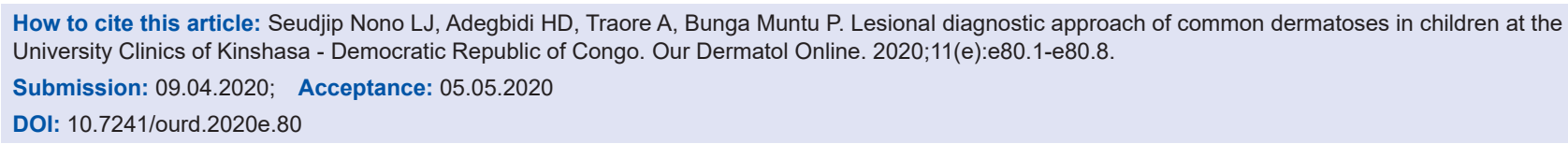




\title{
Approche diagnostique lésionnelle des dermatoses fréquentes chez l'enfant aux Cliniques Universitaires de Kinshasa - République Démocratique du Congo
}

\author{
Lydie Joelle Seudjip Nono', Hugues D. Adegbidi², Adama Traore ${ }^{3}$, Paulo Bunga Muntu
}

${ }^{1}$ Department of Dermatology and Venereolology, Kinshasa University Clinics, Democratic Republic of Congo, ${ }^{2}$ Department of Dermatology and Venereolology, University Hospital Center, Cotonou, Benin, ${ }^{3}$ Department of Dermatology and Venereolology, Yalgado Ouédraogo University Hospital Center in Ouagadougou, Burkina Faso, ${ }^{4}$ Department of Pediatrics, Kinshasa University Clinics, Democratic Republic of Congo

Corresponding author: Dr. Lydie Joelle Seudjip Nono, E-mail: seupiziemi@gmail.com

\section{RÉSUMÉ}

Contexte: Plusieurs auteurs abordent les études épidémiologiques sur les dermatoses suivant une approche diagnostique étiologique; l'école Willaniste étant réservée la plupart du temps aux apprenants en dermatologie et/ou aux médecins non dermatologues. Lobjectif de cette étude est de déterminer le profil des dermatoses chez l'enfant en recourant exclusivement au type de lésion élémentaire. Matériel et méthodes: Dans une étude rétrospective, analytique et descriptive, les données des enfants avec dermatoses suivis dans le Service de Dermatologie des Cliniques Universitaires de Kinshasa entre le ler juin 2009 et le 31 décembre 2011, étaient colligées. Les paramètres d'intérêts comprenaient les caractéristiques épidémiologiques et cliniques. Résultats: La fréquence hospitalière des dermatoses chez l'enfant (DE) selon l'approche diagnostique lésionnelle est de 40,89\% (818/1994). Leur âge médian était de 60 mois (QEI 60$65,9)$ avec une prédominance féminine $(55,7 \%$, sex-ratio de 1,25/1). Les nourrissons étaient plus nombreux $(30,6 \%)$. Les DE prédominaient en saison sèche (54\%). Les types lésionnels intriqué $(21,02 \%)$ et vésiculeux $(20,29 \%)$ étaient les plus nombreux avec respectivement pour chefs de file la teigne du cuir chevelu $(31,9 \%)$ et la dermatite atopique (54,2\%). Les types lésionnels papuleux, érythémateux, tumoral et intriqué étaient liés au sexe et à l'âge; pustuleux et tumoral exclusivement liés à la saison, de façon statistiquement significative $(\mathrm{p}<0,05)$. Prises dans leur globalité, les dermatoses les plus fréquentes étaient la dermatite atopique (11\%), le prurigo strophulus (10,8\%), l'impétigo (7,4\%), Tinea capitis $(6,7 \%)$ et la scabiose (6,4\%). Conclusion: L'importance de ce travail réside dans l'intérêt de la prise en compte des deux approches, lésionnelle et étiologique des dermatoses, pour leur prise en charge globale et des études de recherche. En effet, l'approche lésionnelle également importante dans l'étude des dermatoses, s'avère à notre connaissance pauvre en références dans la littérature.

Mots clés: Dermatoses; Enfants; Diagnostic lésionnel; Kinshasa

\section{INTRODUCTION}

\section{Contexte}

Les dermatoses constituent un motif fréquent de consultation en milieu pédiatrique [1-4].

Celles-ci sont le plus souvent diagnostiquées selon les approches étiologique par les dermatologues [5] et lésionnelle par les apprenants en dermatologie et/ou les médecins non dermatologues qui auraient quelques notions de lésions élémentaires.

Plusieurs auteurs en la matière abordent l'étude épidémiologique des dermatoses en appliquant l'école de William Dubreuillh qui se base sur l'étude de l'étiologie ou de la physiopathologie des maladies de la peau $[1,2,4,6]$, ne nécessitant pas toujours dans l'exercice clinique quotidien une confirmation paraclinique.

\footnotetext{
How to cite this article: Seudjip Nono LJ, Adegbidi HD, Traore A, Bunga Muntu P. Approche diagnostique lésionnelle des dermatoses fréquentes chez l'enfant aux Cliniques Universitaires de Kinshasa - République Démocratique du Congo. Our Dermatol Online. 2020;11(e):e80.1-e80.8.

Submission: 09.04.2020; Acceptance: 05.05.2020

DOI: 10.7241 /ourd.2020e.80
} 
Fort de ce constat, nous nous sommes proposé de mener une étude épidémiologique des dermatoses chez l'enfant en recourant exclusivement à la description des lésions élémentaires, en vue d'émettre un diagnostic du type de dermatose.

Le but de notre étude est de déterminer les principaux types de dermatoses chez l'enfant en milieu hospitalier en recourant à l'approche diagnostique lésionnelle.

\section{MATÉRIEL ET MÉTHODES}

\section{Cadre de l'étude}

La présente étude était réalisée dans le service de dermatologie aux Cliniques Universitaires de Kinshasa, en République Démocratique du Congo (RDC).

2. Type, période et durée d'étude

Il s'agissait d'une étude descriptive et analytique portant sur 818 dossiers des patients âgés de 0 à 18 ans, colligés dans le service de dermatologie - vénéréologie des with Cliniques Universitaires de Kinshasa (CUK) en RDC durant une période de 31 mois (ler juin 2009 au 31 décembre 2011).

3. Population d'étude et échantillon:

818 dossiers des enfants examinés, âgés de 0 à 18 ans, étaient inclus dans l'étude, répondant de manière complète aux variables d'intérêt. N'étaient pas inclus dans l'étude, tous les dossiers incomplets en rapport avec les variables recherchées.

4. Variables étudiées:

Les données répertoriées étaient épidémiologiques (âge, sexe, mois de l'année et saison) et cliniques (groupe lésionnel et diagnostic clinique de la $\mathrm{DE})$. L'âge était réparti de manière suivante: 0-2 ans (nourrissons), 3-5 ans (âge préscolaire), 6-12 ans (âge scolaire) et 13-18 ans (adolescence). La saison était répartie en deux: pluvieuse et sèche. Ainsi toute dermatose diagnostiquée pendant le mois de janvier, février, avril, mai, septembre, octobre, novembre et décembre était considérée apparaître à la saison pluvieuse, par contre pour celles de la saison sèche, il s'agissait des mois de mars, juin, juillet et août [7]. Le diagnostic était basé sur la clinique seule au départ du type de lésion élémentaire.

5. Définitions opérationnelles

Lésion élémentaire: lésion initiale ou secondaire, telle qu'elle apparaît à l'œil nu, grâce à elle on fait un diagnostic immédiat et de là, une classification de la dermatose.
Lésion intriquée: association de plus d’une lésion élémentaire

Groupe lésionnel: ensemble de dermatoses ayant en commun le même type de lésion élémentaire Diagnostic clinique: dermatose objectivée à la fin de l'examen clinique au départ du type de la lésion élémentaire

6. Méthodes techniques et instruments de collecte des données

Les renseignements utiles à l'étude étaient prélevés dans les registres et toutes les fiches de consultation des patients répondant aux normes de l'étude et durant la période retenue à cette fin. Ils étaient consignés ensuite sur les fiches de collecte de données, renfermant toutes nos variables d'étude. Un ordinateur portable de marque hp EliteBook avait permis de compiler ces données dans un logiciel Excel afin de dégager, regrouper et analyser toutes ces variables d'intérêt.

7. Considérations éthiques

La confidentialité et l'anonymat étaient respectés lors de la collecte des données et de la prise des photos, pour lesquelles les yeux étaient voilés du visage.

8. Méthode de traitement des données

Toutes les données étaient saisies et analysées à l'aide du logiciel SPSS (Statistical package for social sciences, Chicago) pour Windows version 21. Le traitement statistique des données avait consisté à calculer les moyennes, l'écart-type, la médiane et l'écart interquartile pour les variables quantitatives et les proportions pour les variables qualitatives. Le test de chi carré de Pearson ou le test exact de Fisher était utilisé pour comparer les proportions selon le cas. Le seuil de signification statistique était fixé à $5 \%$

\section{RÉSULTATS}

\section{Données épidémiologiques}

Sur un effectif de 1994 patients reçus dans le service pendant la période d'étude, 818 étaient des enfants (40,8\%); conformément au tableau 1 , le sexe féminin était prépondérant $(55,7 \%)$ avec un sex ratio femme/homme de 1,25/1. Leur âge médian était de 60 mois (60-65,9 mois), avec des extrêmes 0 à 218 mois. Les nourrissons constituaient la tranche d'âge la plus importante (30,6\%). Les dermatoses prédominaient en saison sèche $(54,0 \%)$ avec des pics aux mois de février $(12,3 \%)$ et de juillet $(11,9 \%)$. 


\section{Données cliniques}

La répartition des types lésionnels des dermatoses en fonction du sexe (Tableau 2) avait montré que les intriqués $(28,01 \%)$ et les vésiculeux $(20,3 \%)$ étaient les plus fréquentes avec prédominance dans le sexe masculin $(28,21 \%, \mathrm{p}<0,001$ pour le type intriqué). Les types lésionnels papuleux $(19,1 \%)$, érythématosquameux $(10,5 \%)$, tumoral $(5,9 \%)$, érythémateux $(4,6 \%)$ et intriqué $(28,01 \%)$ étaient liés au sexe avec $\mathrm{p}<0,05$.

La répartition des types lésionnels des dermatoses en fonction de l'âge. Selon le tableau 3, le type de lésion élémentaire etait lié à l'âge $(\mathrm{p}<0,05)$, à l'exception des

\begin{tabular}{lcc} 
Tableau 1: Données épidémiologiques & \\
\hline Variables & $\mathbf{n}=\mathbf{8 1 8}$ & Pourcentage \\
\hline Sexe & 362 & 44,3 \\
Masculin & 456 & 55,7 \\
Féminin & & \\
Age & 250 & 30,6 \\
0-2 ans & 188 & 23,0 \\
3-5 ans & 215 & 26,3 \\
6-12 ans & 165 & 20,2 \\
13-18 ans & & \\
Saison & 442 & 54,0 \\
Sèche & 376 & 46,0 \\
Pluvieuse & & \\
Mois d'admission & 53 & 6,5 \\
Janvier & 101 & 12,3 \\
Février & 88 & 10,8 \\
Mars & 70 & 8,6 \\
Avril & 55 & 6,7 \\
Mai & 55 & 6,7 \\
Juin & 97 & 11,9 \\
Juillet & 77 & 9,4 \\
Aout & 68 & 8,3 \\
Septembre & 31 & 3,8 \\
Octobre & 51 & 6,2 \\
Novembre & 72 & 8,8 \\
Décembre & & \\
\hline
\end{tabular}

dyschromies, nodules, squames, kératoses, perte de cheveux et lésions non définies. Les types lésionnels vésiculeux étaient plus observés entre $0-2$ ans et $6-$ 12 ans; par contre le type papuleux étaient l'apanage des périodes pré-scolaire et l'adolescence $(\mathrm{p}<0,05)$.

La répartition des types lésionnels en fonction des saisons (Tableau 4) indique que seuls les pustuleux et les tumoraux étaient liés à la saison de façon statistiquement significative.

En termes de fréquences, la répartition des types lésionnels des dermatoses de l'enfant (Tableau 5) avait montré que la dermatite atopique $(52,2 \%)$ était la plus fréquente dans le groupe des dermatoses vésiculeuses; le prurigo strophulus $(57,1 \%)$ dans le groupe papuleux, le pityriasis rosé de Gibert (39,5\%) pour le type érthématosquameux, l'impétigo $(60,4 \%)$ pour le type pustuleux; les cicatrices hypertrophiques $(31,3 \%)$ pour le type tumoral; l'érythème fessier en $\mathrm{W}$ $(63,1 \%)$ pour le type érythémateux; le vitiligo $(78,1 \%)$ pour le type dyschromique et tinea capitis $(31,9 \%)$ pour le type intriqué.

Conformément au tableau 6, cinq diagnostics s'avéraient prépondérants avec la dermatite atopique et le prurigo strophulus en tête de file, suivis de l'impétigo, du tinea capitis et de la scabiose.

\section{DISCUSSION}

\section{Données épidémiologiques}

Le but de la présente étude était de déterminer l'incidence des dermatoses chez l'enfant (DE) selon l'approche diagnostique lésionnelle, au service de dermatologie - vénéréologie des CUK.

Tableau 2: Type lésionnel selon le sexe

\begin{tabular}{|c|c|c|c|c|}
\hline Type lésionnel & Tous $\mathrm{n}=818$ & Masculin $n=365$ & Féminin $n=453$ & $p$ \\
\hline Vésiculeux & $166(20,3)$ & $77(21,3)$ & $89(19,5)$ & 0,297 \\
\hline Papuleux & $156(19,1)$ & $53(14,6)$ & $103(22,6)$ & 0,003 \\
\hline Erythématosquameux & $86(10,5)$ & $25(6,8)$ & $61(13,4)$ & 0,001 \\
\hline Pustuleux & $101(12,3)$ & $43(11,9)$ & $58(12,7)$ & 0,400 \\
\hline Tumoral & $48(5,9)$ & $13(3,6)$ & $35(7,7)$ & 0,009 \\
\hline Erythémateux & $38(4,6)$ & $24(6,5)$ & $14(3,0)$ & 0,011 \\
\hline Dyschromique & $33(4,0)$ & $17(4,7)$ & $16(3,5)$ & 0,248 \\
\hline Bulleux & $3(0,4)$ & $3(0,8)$ & $0(0,0)$ & - \\
\hline Nodulaire & $1(0,1)$ & $0(0,0)$ & $1(0,2)$ & - \\
\hline Intriqué & $172(28,01)$ & $103(28,21)$ & $69(15,2)$ & $<0,001$ \\
\hline Squameux & $7(0,8)$ & $3(0,82)$ & $4(0,88)$ & 0,395 \\
\hline Kératosique & $2(0,2)$ & $1(0,27)$ & $1(0,22)$ & 0,779 \\
\hline Perte de cheveux & $3(0,3)$ & $1(0,27)$ & $2(0,44)$ & 0,607 \\
\hline Pas de lésion élémentaire & $2(0,2)$ & $2(0,5)$ & $0(0,0)$ & 0,617 \\
\hline
\end{tabular}


Tableau 3: Répartition des types lésionnels selon l'âge

\begin{tabular}{|c|c|c|c|c|c|}
\hline \multirow[t]{2}{*}{ Type lésionnel } & \multicolumn{4}{|c|}{ Age } & \multirow[t]{2}{*}{$p$} \\
\hline & $0-2$ ans $n=251$ & 3-5 ans $n=192$ & $6-12$ ans $n=212$ & $13-18$ ans $n=163$ & \\
\hline Vésiculeux & $69(27,6)$ & $34(17,7)$ & $46(21,6)$ & $17(10,4)$ & $<0,001$ \\
\hline Papuleux & $35(14,0)$ & $44(22,9)$ & $38(17,9)$ & $39(23,9)$ & 0,029 \\
\hline $\begin{array}{l}\text { Erythémato } \\
\text { squameux }\end{array}$ & $22(8,8)$ & $12(6,2)$ & $21(9,9)$ & $31(19,0)$ & 0,002 \\
\hline Pustuleux & $37(14,8)$ & $22(11,4)$ & $12(5,6)$ & $30(18,4)$ & 0,001 \\
\hline Tumoral & $22(8,8)$ & $9(4,6)$ & $14(6,6)$ & $3(1,8)$ & 0,001 \\
\hline Erythémateux & $33(13,1)$ & $4(2,0)$ & $0(0,0)$ & $1(0,6)$ & $<0,001$ \\
\hline Dyschromique & $4(1,5)$ & $8(4,1)$ & $11(5,1)$ & $10(6,1)$ & 0,068 \\
\hline Bulleux & $3(1,1)$ & $0(0,0)$ & $0(0,0)$ & $0(0,0)$ & - \\
\hline Nodulaire & $0(0,0)$ & $0(0,0)$ & $0(0,0)$ & $1(0,6)$ & - \\
\hline Intriqué & $26(10,35)$ & $53(27,6)$ & $64(30,18)$ & $29(17,7)$ & $<0,0001$ \\
\hline Squameux & $0(0,0)$ & $3(1,5)$ & $4(1,88)$ & $0(0,0)$ & 1 \\
\hline Kératosique & $0(0,0)$ & $1(0,5)$ & $1(0,4)$ & $0(0,0)$ & 0,480 \\
\hline Perte de cheveux & $0(0,0)$ & $2(1,0)$ & $1(0,4)$ & $0(0,0)$ & 1 \\
\hline Pas de lésion élémentaire & $0(0,0)$ & $0(0,0)$ & $0(0,0)$ & $2(1,2)$ & - \\
\hline
\end{tabular}

Tableau 4: Répartition des types lésionnels selon la saison

\begin{tabular}{|c|c|c|c|}
\hline Groupes lésionnels & Saison sèche $n=454$ & Saison pluvieuse $n=364$ & $p$ \\
\hline Vésiculeux & $91(20,0)$ & $75(20,6)$ & 0,445 \\
\hline Papuleux & $81(17,4)$ & $75(20,6)$ & 0,309 \\
\hline Erythématosquameux & $52(11,4)$ & $34(9,3)$ & 0,147 \\
\hline Pustuleux & $41(9,0)$ & $60(16,4)$ & 0,003 \\
\hline Tumoral & $35(7,7)$ & $13(3,5)$ & 0,005 \\
\hline Erythémateux & $19(4,1)$ & $18(4,9)$ & 0,156 \\
\hline Dyschromique & $19(4,1)$ & $14(3,8)$ & 0,408 \\
\hline Bulleux & $3(0,6)$ & $0(0,0)$ & - \\
\hline Nodule & $0(0,0)$ & $1(0,2)$ & - \\
\hline Intriqué & $102(26,4)$ & $64(17,5)$ & 0,288 \\
\hline Squameux & $7(1,5)$ & $0(0,0)$ & 0,445 \\
\hline Kératosique & $1(0,2)$ & $1(0,2)$ & - \\
\hline Perte de cheveux & $1(0,2)$ & $2(0,5)$ & - \\
\hline Pas de lésions élémentaires & $2(0,4)$ & $0(0,0)$ & - \\
\hline
\end{tabular}

La fréquence des DE dans cette étude était de 40,8\%, constat semblable au Yemen [8] et en Egypte [9], respectivement $45,1 \%$ et $40 \%$, contrairement aux observations maliennes $(31,51-32,9 \%)[2,10]$. Le sexe féminin était majoritaire $(55,7 \%)$ avec un sex ratio $\mathrm{F} / \mathrm{H}$ de 1,25 . Nos résultats s'accordent avec ceux observés à l'Est de la RDC [11] mais divergent de ceux des études Nigériane [12] et Turque [13] où les enfants en période d'âge scolaire étaient plus nombreux. La prédominance des dermatoses chez les nourrissons pourrait s'expliquer par la précarité des conditions de vie des populations kinoises en général et des confins des CUK en particulier; les réalités démographiques pourraient justifier la grande fréquence du sexe féminin [4].

La saison sèche était l'alliée des DE dans $54 \%$ des cas avec des pics élevés en février $(12,3 \%)$ et en juillet $(11,9 \%)$. Dans une étude de Seudjip et al. [4] portant sur le profil des groupes étiologiques des DE, les observations sont les mêmes et s'accordent avec celles de Tamer et al. en Turquie [13], mais divergent de celles de El Khateeb et al. en Egypte [9]. Le climat sec et froid de la saison sèche favorise d'une part la sécheresse cutanée, grande pourvoyeuse des irritations et des effractions cutanées, par conséquent de la survenue des dermatoses, et d'autre part la malpropreté corporelle à cause de la diminution de la fréquence de bain corporel.

\section{Données cliniques}

Les dermatoses intriquées $(28,01 \%)$, vésiculeuses $(20,3 \%)$ et papuleuses $(19,1 \%)$ étaient les plus fréquentes dans le présent travail (Tableau 2). La survenue des DE était liée au sexe (p inférieur $(\mathrm{p}<0,05$; Tableau 2) pour les types lésionnels intriqué (tinea capitis: 31,9\%; scabiose: 30,8\%), érythémateux (Erythème Fessier du nourrisson en W: 63,1\%), tumoral (cicatrice hypertrophique: $31,3 \%$ ), érythémato-squameux (Pityriasis Rosé de Gibert: 39,5\%) et papuleux (prurigo strophulus: 67,1\%) (Tableau 5). 
Tableau 5: Répartition de dermatoses selon les types lésionnels

\begin{tabular}{|c|c|c|}
\hline Dermatoses par groupe lésionnel & $\mathbf{n}$ & $\%$ \\
\hline Intriqué & 172 & 100,0 \\
\hline Tinea capitis & 55 & 31,9 \\
\hline Scabiose & 53 & 30,8 \\
\hline Tinea corporis & 25 & 15,5 \\
\hline Myase rampante & 9 & 5,2 \\
\hline Onychomycose & 3 & 1,7 \\
\hline Myase furonculoïde & 1 & 0,5 \\
\hline Tungose & 1 & 0,5 \\
\hline A déterminer & 9 & 5,2 \\
\hline Ongle incarné & 4 & 2,3 \\
\hline Erysipèle & 1 & 0,5 \\
\hline Sclérodermie & 1 & 0,5 \\
\hline Lèpre & 1 & 0,5 \\
\hline Bébé collodion & 1 & 0,5 \\
\hline Type Vésiculeux & 166 & 100,0 \\
\hline Dermatite atopique & 90 & 54,2 \\
\hline Dermatide de contact & 17 & 10,2 \\
\hline Sudamina & 46 & 27,7 \\
\hline Zona & 2 & 1,2 \\
\hline Herpès & 5 & 3,0 \\
\hline Varicelle & 6 & 3,6 \\
\hline Papuleux & 156 & 100,0 \\
\hline Urticaire & 28 & 17,9 \\
\hline Prurigo strophulus & 89 & 57,1 \\
\hline Erythéme polymorphe & 5 & 3,2 \\
\hline Granulome anulaire & 3 & 1,9 \\
\hline Lichen plan & 7 & 4,5 \\
\hline Epidermodysplasie verruciforme & 2 & 1,3 \\
\hline Verrue vulgaire & 17 & 10,9 \\
\hline Keratose pilaire & 4 & 2,6 \\
\hline Piryriasis rubra pillaire & 1 & 0,6 \\
\hline Pustuleux & 101 & 100,0 \\
\hline Impétigo & 61 & 60,4 \\
\hline Acné vulgaire & 31 & 30,7 \\
\hline Foliculite & 9 & 8,9 \\
\hline Erythémato-squameux & 86 & 100,0 \\
\hline Pityriasis rosé de Gibert & 34 & 39,5 \\
\hline Eczématide & 23 & 26,7 \\
\hline Dermite séborrhéïque & 11 & 12,7 \\
\hline Pytiriasis versicolor & 10 & 11,6 \\
\hline Psoriasis & 4 & 4,6 \\
\hline Tinea corporis & 1 & 1,1 \\
\hline A determiner & 2 & 2,3 \\
\hline Tumoral & 48 & 100,0 \\
\hline Cicatrice hypertrophique & 15 & 31,3 \\
\hline Hémangiome infantile & 14 & 29,2 \\
\hline Molluscum contagesum & 8 & 16,7 \\
\hline Naivus verruqueux & 3 & 6,3 \\
\hline Condylome anogénital & 2 & 4,2 \\
\hline Neurofibromatose & 2 & 4,2 \\
\hline Maladie de Kaposi & 2 & 4,2 \\
\hline Condylome buccal & 1 & 2,1 \\
\hline Botriomycome & 1 & 2,1 \\
\hline Erythémateux & 38 & 100,0 \\
\hline Toxidermie & 8 & 21,0 \\
\hline Erythème fessier en $\mathrm{W}$ & 24 & 63,1 \\
\hline Erythème fessier en $Y$ & 6 & 15,7 \\
\hline Dyschromique & 33 & 100,0 \\
\hline Vitiligo & 25 & 78,1 \\
\hline Hyperpigmentation séquellaire & 7 & 21,2 \\
\hline
\end{tabular}

Tableau 5: (Continued)

\begin{tabular}{llc}
\hline Dermatoses par groupe lésionnel & $\mathbf{n}$ & $\%$ \\
\hline Mosaicisme & 1 & 3,1 \\
Squameux & 7 & 100,0 \\
Icthtyose vulgaire & 7 & 4,0 \\
Kératosique & 2 & 100,0 \\
Kératodermie palmo-plantaire & 2 & 1,1 \\
Perte de cheveux & 3 & 100,0 \\
Pélade & 3 & 1,7 \\
Pas de lésions élémentaires & 2 & 100,0 \\
Gonococcie & 2 & 1,1 \\
\hline
\end{tabular}

Tableau 6: Diagnostics les plus fréquents

\begin{tabular}{lcc}
\hline Diagnostics & $\mathbf{n}$ & $\%$ \\
\hline Dermatite atopique & 90 & 11 \\
Prurigo strophulus & 89 & 10,8 \\
Impétigo & 61 & 7,4 \\
Tinea capitis & 55 & 6,7 \\
Scabiose & 53 & 6,4 \\
Sudamina & 46 & 5,6 \\
Pityriasis rosé de Gibert & 34 & 4,1 \\
Acné vulgaire & 31 & 3,7 \\
Urticaire & 28 & 3,4 \\
Vitiligo & 25 & 3,0 \\
Tinea corporis & 25 & 3,0 \\
Erythème fessier en W & 24 & 2,9 \\
Eczématide & 23 & 2,8 \\
Verrue vulgaire & 17 & 2,0 \\
Dermatite de contact & 17 & 2,0 \\
\hline
\end{tabular}

Sans tenir compte de la classification des DE selon l'approche lésionnelle par d'autres auteurs, tinea capitis et scabiose se trouvaient être les plus fréquentes des dermatoses intriquées et représentaient sur l'effectif global de l'étude respectivement $6,7 \%$ et $6,4 \%$. En comparant ce constat diagnostique à ceux des travaux similaires mais selon l'approche étiologique, il y'a un accord avec Seudjip et al. [4] mais une contradiction avec Seudjip et al. [14], où la scabiose représentait $30,6 \%$ et tinea capitis 3,8\% de l'effectif global des dermatoses infectieuses.

Les types lésionnels étaient liés à l'âge pour les dermatoses intriquées, érythémateuses, tumorales, pustuleuses, érythémato-squameuses, papuleuses et vésiculeuses (Tableau 3). Tinea capitis et la scabiose étaient l'apanage de la tranche d'âge de 3 à 12 ans $(\mathrm{p}<0,0001)$. Pour tinea capitis,la présente étude va dans le même ordre d'idées que Seudjip et al. [4,14] et Fofana et al. [2]. Quant à la scabiose, nos résultats confortent ceux de Seudjip et al. [14], bien que leur étude portait exclusivement sur les dermatoses infectieuses chez l'enfant.

La survenue de la gale et de la teigne du cuir chevelu durant la tranche d'âge de $3-12$ ans pourrait 
s'expliquer par la phase de croissance que traversent les enfants à cet âge, le contact important interhumain et avec l'environnement $[14,15]$, en plus du bas niveau socio-économique [16].

Les dermatoses vésiculeuses étaient liées au sexe $(\mathrm{p}<0,001)$, avec un pic élevé chez les nourrissons $(27,6 \%)$ (Tableau 3$)$ et la dermatite atopique était la plus fréquente $(54,2 \%)$ (Tableau 5 ). Toujours prises sous l'angle étiologique, plusieurs auteurs observent les dermatoses vésiculeuses comme étant les plus nombreuses chez les nourrissons [17-19]. La prédominance de la dermatite atopique dans cette étude s'accorde avec le constat de Seudjip et al. [4,11] .

L'hygiène précaire de la peau du nourrisson dans notre milieu, associée au survêtement en région tropicale, peuvent justifier entre autre la prépondérance des dermatoses vésiculeuses. Lurbanisation, la croissance progressive de la pollution dans les grandes métropoles et la xérose cutanée due au climat froid et sec de la saison sèche pourraient justifier la prépondérance de la dermatite atopique [4].

Les dermatoses papuleuses venaient en $3^{\text {ème }}$ position avec 19,1\% des cas (Tableau 2); elles étaient liées à l'âge $(\mathrm{p}=0,029)$ avec des pics élevés entre 3-5 ans $(22,9 \%)$ et $13-18$ ans $(23,9 \%)$ (Tableau 3$)$; le prurigo strophulus se trouvant être le diagnostic le plus fréquent dans ce groupe $(57,1 \%)$ (Tableau 5$)$.

Ce constat se rapproche de ceux de plusieurs auteurs $[4,20]$ pour la tranche d'âge de $3-5$ ans contrairement à l'observation de Lenga et al. [21] où la prédominance se situe entre $6-8$ ans.

Les piqûres d'insectes, le port des vêtements non couvrant, le non assainissement et la pollution de l'environnement pourraient étayer la survenue accrue du prurigo strophulus chez les enfants [1, 21, 22].

Le relevé des diagnostics (Tableau 6) montre que les dix DE les plus fréquentes étaient identiques selon les approches diagnostiques étiologique et lésionnelle et ce, aux fréquences comparables [4].

\section{CONCLUSION}

Limportance du présent travail réside dans l'intérêt de la prise en compte des deux approches, tant lésionnelle qu'étiologique, pour la prise en charge globale des dermatoses. En effet, l'approche lésionnelle pourtant également importante dans l'étude des dermatoses, s'avère à notre connaissance pauvre en références dans la littérature.

\section{Remerciements}

Notre gratitude est exprimée au Docteur Aliocha Nkodila pour les analyses statistiques.

\section{Statement of Human and Animal Rights}

All procedures followed were in accordance with the ethical standards of the responsible committee on human experimentation (institutional and national) and with the Helsinki Declaration of 1975, as revised in 2008 .

\section{Statement of Informed Consent}

Informed consent was obtained from all patients for being included in the study.

\section{RÉFÉRENCES}

1. Kourouma HS, Kouassi Yi, Ecra EJ, Kaloga M, Gbery iP, Ahogo C, et al. Dermatoses de l'enfant: panorama des entités cliniques en consultation à Abidjan. Rev Int Sc Méd. 2017;19:144-8.

2. Youssouf F, Bekaye T, Adama D, Ousmane F, Siritio B, Lamissa C, et al. Profil épidémio-clinique des dermatoses chez les enfants vus en consultation dermatologique dans le service de dermatologie du centre national d'appui à la lutte contre la maladie à Bamako (Mali). Pan Afr Med J. 2016;25:238.10564.

3. Traoré A, Kouéta F, Sanou I, Kam K, Dao L, Barro F, et al. Les dermatoses courantes de l'enfant dans un service de dermatologie en milieu tropical. Service de Coopération et d'Action Culturelle éd, Ouagadougou. http://www.chu-rouen.fr/chnpo/Annales/ Pubped9.html. Consulté le 17 juillet 2018.

4. Seudjip NLJ, Traore A, Mazebo PS, Bunga MP. Profile of the Etiological Groups of Child Dermatosis at the University Clinics of Kinshasa - Democratic Republic of the Congo. Curr Updates Dermatol Probl. 2019;2019:-11.

5. Wallach D. Guide pratique de dermatologie, 2è éd., Mimi et Masson.

6. Emodi IJ, Ikefuna AN, Uchendu U. Skin diseases among children attending the outpatient clinic of the University of Nigeria teaching hospital, Enug Afr Health Sci. 2010;10:362-6.

7. Mulumba MP, Le paludisme de l'enfant à Kinshasa (Zaire): Influence de saison, de l'âge, de l'environnement et du standing familial. Méd Trop. 1990;50:53-4.

8. Lal Khatri M. Spectrum of skin diseases in Yemen (Hajjah and adjacent region). Int J Dermatol. 2004;43:580-1.

9. El-Khateeb EA. The spectrum of paediatric dermatoses in a university hospital in Cairo, Egypt. J Eur Acad Dermatol Venereol. 2011;25:666-72.

10. Mahe A, Cisse IA, Faye O, Ndiaye HT, Niamba P. Skin diseases in Bamako (Mali). Int J Dermatol. 1998;37:673-6.

11. Semikenke S, Adégbidi H, Minani J, Bisimwa G. Les dermatoses de l'enfant en milieu hospitalier à Bukavu: aspects épidémiologiques et cliniques. Ann Dermatol Vénéréol. 2018;145:40.

12. Ayanlowo O, Puddicombe O, Gold-Olufadi S. Pattern of skin diseases among children attending a dermatology clinic in Lagos, Nigeria. Pan Afr Med J. 2018;29:162. 


\section{www.odermatol.com}

13. Tamer E, Ilhan MN, Polat M, Lenk N, Alli N. Prevalence of skin diseases among pediatric patients in Turkey. J Dermatol. 2018;35:413-8.

14. Seudjip Nono LJ, Kakiesse Musumba V, Mazebo MPku S, Kasongo Mulenda F, Tshilombo Mwindila JM, Mbanzulu Dimbu C, et al. Dermatose infectieuse chez les enfants fréquentant un établissement de santé à Kinshasa/République Démocratique du Congo. J Innovat Res Health Scien Biotechnol. 2018;3:639-45.

15. Kouotou EA, Fokoua DCM, Kechia FA, Somo MR. Teigne du cuir chevelu: profil épidémiologique en milieu scolaire camerounais. Ann Dermatol Vénéréol. 2016;143:42.

16. Seudjip NLJ, Kakiesse MV, Musibwe A, Kasongo MF, Tshilombo MJM, Mutombo TM, et al. Panorama des dermatoses infectieuses aux Cliniques Universitaires de Kinshasa, en République Démocratique du Congo. Ann Afr Med. 2018;11:e3009-17.

17. Shahram B, Shahram Z, Abdoul-Ali M. Report: Skin disease patterns in Hormozigan, Iran MD. Int J Dermatol. 2005;44:641-5.

18. Furue M, Yamazaki S, Jimbow K, Tsuchida T, Amagai M, Tanaka T, et al. Prevalence of dermatological disorders in Japan: A nationwide, cross-sectional, seasonal, multicenter, hospital-based study. J Dermatol. 2011;38:310-20.

19. Andonaba J, Barro-Traoré F, Diallo B, Sakana L, Niamba P,
Traoré A. Aspects épidémiologiques des affections dermatologiques au Centre hospitalier universitaire de Souro Sanou de BoboDioulasso. Ann Afr Med. 2010;4:668-77.

20. Ahogo C, Sangare A, Kassi K, Ecra E, Kaloga M. Le prurigo strophulus: Aspects épidémiologiques et étiologiques sur peau noire à Abidjan (Côte d'Ivoire). Rev Int Sc Méd. 2007;9:29-33.

21. Lenga A, Lenga - Loumingou A, Mabika Moussounda M, VouidibioJ. The Prurigo Strophulus in Brazzaville: Demonstration of Vectors and Study of Some Associated Bioecological Parameters. Pakistan J Zool. 2013;45:121-8.

22. Adegbidi H, Degboé B, Saka B, Elegbedé A, Atadokpedé F. Profil des dermatoses immunoallergiques chez les enfants dans le service de dermatologie du CNHU-C (Bénin). Méd Santé Trop. 2014;24:446-8.

Copyright by Lydie Joelle Seudjip Nono, et al.. This is an open access article distributed under the terms of the Creative Commons Attribution License, which permits unrestricted use, distribution, and reproduction in any medium, provided the original author and source are credited.

Source of Support: Nil, Conflict of Interest: None declared. 